\title{
Feasibility and accuracy of fetal echocardiography at routine 11 to 13 weeks 6 days ultrasound performed on unselected obstetric population in a tertiary maternity centre with high patient turnover
}

\author{
Vidyashree G. Poojari, Arevidya Reddy, Akhila Vasudeva*
}

\begin{abstract}
Department of Obstetrics and Gynecology, Kasturba Medical College, Manipal Academy of Higher Education,
\end{abstract} Manipal, Karnataka, India

Received: 11 December 2017

Accepted: 19 December 2017

\section{*Correspondence:}

Dr. Akhila Vasudeva,

E-mail: akhilavasudeva@gmail.com

Copyright: (C) the author(s), publisher and licensee Medip Academy. This is an open-access article distributed under the terms of the Creative Commons Attribution Non-Commercial License, which permits unrestricted non-commercial use, distribution, and reproduction in any medium, provided the original work is properly cited.

\section{ABSTRACT}

Background: Congenital heart defects (CHD) remain the most prevalent congenital malformations, with an estimated prevalence of 6-8 per 1000 live births. Routinely, fetal echo is performed around 18-22 weeks among high risk cases. A dedicated early fetal echo (EFEC) performed by experts between 11-16 weeks is emerging as an excellent screening tool among high risk cases. However, feasibility of obtaining such cardiac views during routine Nuchal Translucency scan by trained obstetricians, is yet to be known as there is limited time available with high patient turn over. Present study aimed to know the feasibility and accuracy of EFEC in unselected obstetric population in a tertiary maternity centre with high patient turnover.

Methods: This was an observational cohort study from March 2015 to April 2017 at a tertiary maternity referral hospital. Singleton pregnant women undergoing routine 11-13+6 weeks scanning (NT scan) were recruited in the study including both high and low risk pregnancies. Possibility of obtaining 4 basic cardiac views within this limited time was assessed.

Results: A total of 180 early fetal ECHO's were performed during the course of this study. The 4-chamber view $(79.44 \%)$ of the heart was the easiest parameter to visualize. RVOT (28.88\%) was the most difficult view to be obtained. Complete visualization of all the cardiac views was possible in only 34 women (18.88\%). The visualization rates of the cardiac views improved as the gestational age increased. TVS was essential to obtain cardiac views in 5 cases out of $138(3.6 \%)$ in 12-13+6 scans. Whereas, TVS was essential to visualize cardiac views in 34 cases out of $42(80.95 \%)$ between 11 weeks to 11 weeks 6 days. A total of 7 cardiac anomalies were detected during this study. There were 3 major and 4 minor cardiac malformations. Out of the 3 major anomalies, 2 were AVSDs which were diagnosed by EFEC. Minor defects were undetected.

Conclusions: It is feasible to get the basic cardiac views in the limited time available to perform NT scan among unselected obstetric population in a tertiary maternity centre with a high turnover. Gestational age of 12 weeks and above seems to increase the accuracy. TVS is a useful adjunct throughout 11-14 weeks scan.

Keywords: Accuracy, Early Fetal Echocardiogram (EFEC), Feasibility, Nuchal translucency (NT)

\section{INTRODUCTION}

Congenital heart defects (CHD) remains the most prevalent congenital malformation, with an estimated prevalence of 6-8 per 1000 live births. ${ }^{1}$ Prenatal diagnosis of CHD has been shown to have a significant effect on prenatal and postnatal management and outcomes. In addition to the potential medical benefits, fetal diagnosis 
allows for valuable parental counseling, which allows families to make decisions regarding the continuation of pregnancy, and to prepare emotionally for the birth of the child with significant CHD. Accurate prenatal diagnosis may require additional testing of the fetus, including detailed anatomical survey to rule out any other structural deformities and genetic evaluation, which can yield valuable information in overall assessment of the fetus. ${ }^{2}$

Fetal echocardiography remains the gold standard for identification of CHD among at risk pregnancies. In various low-risk as well as unselected groups, fetal echocardiography has excellent sensitivity at picking up major defects. ${ }^{1}$

Referral for fetal echocardiogram typically occurs between 18 and 22 weeks of gestational age. Advances in ultrasound technology has allowed for the significant improvements in high resolution imaging necessary to visualize the developing fetal heart, fetal echocardiography can now be performed in the late first trimester and early second trimester of pregnancy. Despite the known feasibility, accuracy, and potential benefits of early cardiac imaging, it is not the standard of practice in most institutions of the developing world.

Fetal cardiac ultrasound analysis performed between the 12 th and 16th week of pregnancy (compared with the usual 18-22 weeks) is defined as Early Fetal Echocardiography (EFEC). ${ }^{3}$

If the ultrasound is being performed solely for the purpose of fetal ECHO by dedicated fetal cardiologist on a high-risk population, the success rate of achieving all the cardiac views is very high. However, our objective was to test whether along with Nuchal Translucency (NT) scan and anatomical survey during a routine check-up between 11 weeks to 13 weeks 6 days, what would be the possibility of obtaining the cardiac views. We compared the efficacy of Transabdominal (TAS) and Transvaginal (TVS) scan in doing EFEC and also to determine the appropriate gestational age for early fetal echocardiography.

\section{METHODS}

This was an observational cohort study from March 2015 to April 2017 at a tertiary maternity referral hospital. Singleton pregnant women undergoing routine 11-13+6 weeks scanning (NT scan) were recruited in the study. The study included both low risk unselected population as well as those considered to be at high risk of having fetal cardiac anomaly. In the present study, early fetal echocardiogram (EFEC) was defined as study completed between 11 and 13 weeks 6 days of gestational age. We have dedicated 5-10 extra minutes to obtain cardiac views in addition to NT and early anatomical survey. Our aim was to assess the possibility of obtaining 4 basic cardiac views within this limited time (4 chamber view with 2 parallel in flows, $\mathrm{X}$ sign which is crossing over of two outflow tracts, and v/tick sign which is the 3-vessel trachea view).

Cases were informed regarding the limitations of the screening method at early gestational age and also recommended to have a further scan between 18-22 weeks for a more complete diagnosis and informed written consent were taken from all subjects. The study was approved by Institutional Ethical Committee.

$2 \mathrm{D}$ scan was done in women with gestational age ranging between 11 weeks to 13 weeks +6 days i.e. those with crown-rump length between $45-84 \mathrm{~mm}$ were included in the study. All ultrasounds were performed with a 4 to 7 $\mathrm{MHz}$ abdominal transducer, a 4 to $8 \mathrm{MHz}$ transvaginal transducer, or both to optimize views. All EFECs were performed by the single operator who was trained in early anatomical survey, standard fetal echocardiography and NT scan.

The success rate of visualization was expressed as percentages among the various gestational ages and cumulatively. All these women underwent fetal ECHO at 18-22 weeks.

All the neonates born in the institution received care at birth and till 3 days of life by a neonatologist. Neonatal ECHO was done for cases that were either diagnosed to have cardiac anomaly antenatally or in cases with a murmur or suspected cardiac anomaly in the neonate which was not diagnosed antenatally.

The accuracy of the scan done at 11-13 weeks 6 days was compared as against the fetal ECHO done between 18-22 weeks and neonatal ECHO in case of a suspected cardiac disease.

\section{RESULTS}

A total of 180 early fetal ECHO's were performed during the course of this study. 159 women delivered at our institution and 5 underwent medical termination of pregnancy (MTP). The remaining 16 women (8.88\%) were lost to follow up.

Table 1: Success rate of cardiac examination between 11 weeks to 13 weeks 6 days $(N=180)$.

\begin{tabular}{|lll|}
\hline Views & $\begin{array}{l}\text { Number of } \\
\text { cases visualized } \\
\text { N=180 }\end{array}$ & $\begin{array}{l}\text { Percentage of } \\
\text { cases visualized }\end{array}$ \\
\hline 4 chambers & 143 & 79.44 \\
\hline 3 vessels & 123 & 68.33 \\
\hline LVOT & 85 & 47.22 \\
\hline RVOT & 52 & 28.22 \\
\hline All 4 views & 34 & 18.88 \\
\hline None & 29 & 16.11 \\
\hline
\end{tabular}

As shown in Table 1, the 4-chamber view (79.44\%) of the heart was the easiest parameter to visualize. RVOT 
(28.88\%) was the most difficult view to be obtained. Complete visualization of all the cardiac views was possible in only 34 women $(18.88 \%)$. None of the views were visualised in $16 \%$ of the cases (Figure 1).

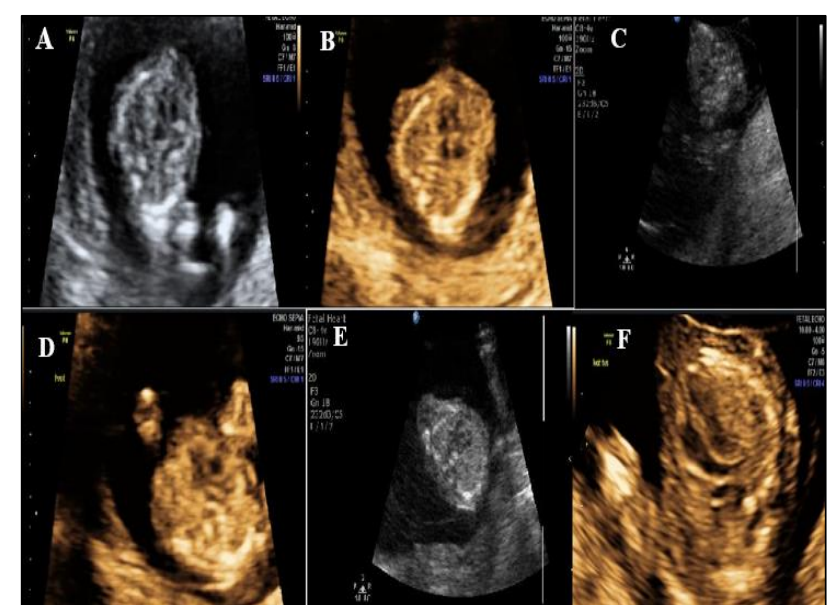

A: TAS showing 4 chamber view, leading to origin of aorta; B: TVS showing the 4-chamber view; C: TAS showing 3 vessel view; D: TVS showing LVOT; E: TAS showing RVOT; F: TVS showing RVOT, leading to 3 vessel view

\section{Figure 1: Normal cardiac views in TAS and TVS between 11 to $13+6$ weeks.}

The visualization rates of the cardiac views improved as the gestational age increased as shown in Table 2 and Figure 2. TVS was used to enhance the visualization of cardiac views in 105 out of 138 cases (76\%) between 12 weeks to 13 weeks 6 days. TVS was essential to obtain cardiac views in 5 cases out of $138(3.6 \%)$ in $12-13+6$ scans. Whereas, TVS was essential to visualize cardiac views in 34 cases out of $42(80.95 \%)$ between 11 weeks to 11 weeks 6 days.

Table 2: Success rate of cardiac examination at 11-11 week +6 days, 12-12 + 6 days, 13-13 + 6 days of gestational age.

\begin{tabular}{|llll|} 
Views & $\begin{array}{l}\text { At 11-11 } \\
\text { weeks + 6 } \\
\text { days } \\
\text { N=42(\%) }\end{array}$ & $\begin{array}{l}\text { At 12-12 } \\
\text { weeks + 6 } \\
\text { days } \\
\text { N= 82 }(\%)\end{array}$ & $\begin{array}{l}\text { At 13-13 } \\
\text { weeks + 6 } \\
\text { days } \\
\text { N=56(\%) }\end{array}$ \\
\hline 4 chambers & $28(66.66)$ & $64(78.04)$ & $51(91.07)$ \\
\hline 3 vessels & $24(57.14)$ & $60(71.42)$ & $39(69.64)$ \\
\hline LVOT & $15(35.71)$ & $35(42.68)$ & $35(62.50)$ \\
\hline RVOT & $12(28.57)$ & $21(25.61)$ & $19(33.92)$ \\
\hline All 4 views & $6(14.28)$ & $16(19.51)$ & $12(21.42)$ \\
\hline None & $11(26.19)$ & $12(14.63)$ & $6(10.71)$ \\
\hline
\end{tabular}

A total of 7 cardiac anomalies were detected during the course of this study. There were 3 major and 4 minor cardiac malformations. Out of the 3 major anomalies, 2 were AVSDs which were diagnosed by EFEC (Figure 3). A case of visceral heterotaxy with completely malrotated heart with TGA and VSD was missed. In this case, the complex cardiac anomaly was very difficult to diagnose with antenatal scan at 18 weeks, due to malrotation of the heart. Fetal ECHO at 18 weeks was also inconclusive of the definitive diagnosis although there was complex cardiac anomaly incompatible with life. Hence couple went ahead with medical termination of pregnancy. Autopsy revealed visceral heterotaxy.

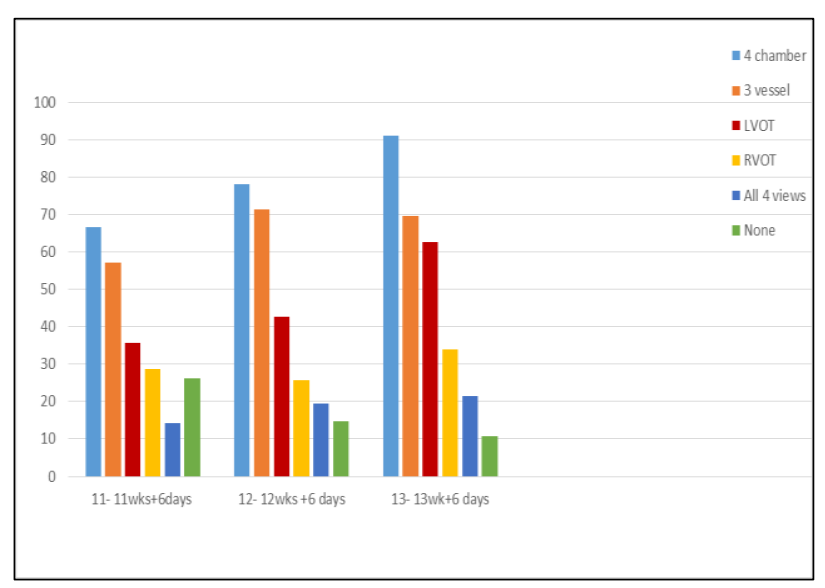

Figure 2: Graphical representation of visibility of cardiac views at different gestational ages.

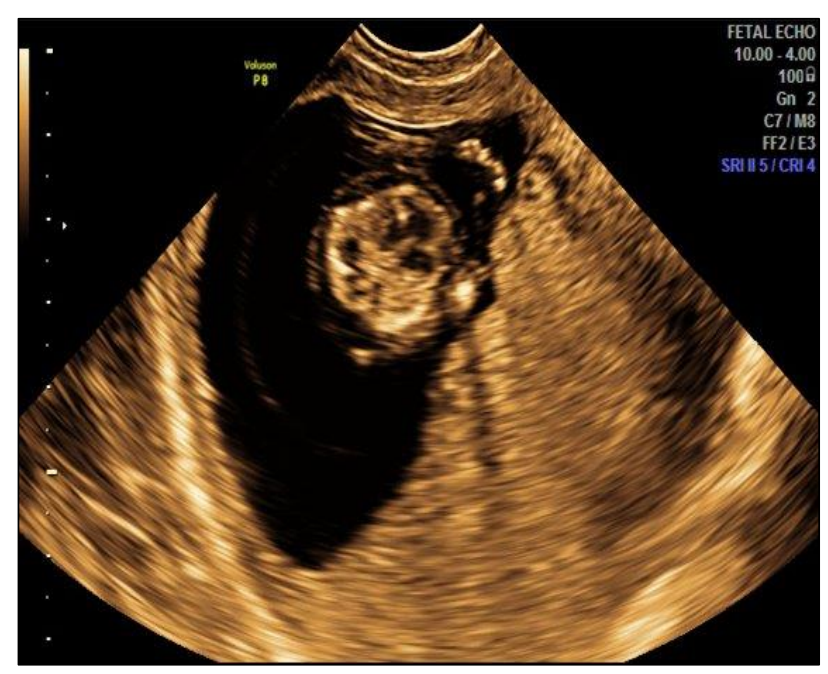

Figure 3: TVS showing AVSD.

None of the 4 minor cardiac defects were detected by the EFEC. They were, two small muscular VSDs, one ASD, and one PDA.

\section{DISCUSSION}

Usual indications for a dedicated EFEC are known or pre-existing risk factors for CHD like mother with overt diabetes, increased NT, major malformations in the early anomaly scan, previous child with complex cardiac anomaly, or parents having a cardiac anomaly. Apart from these indications, no attempts are generally made to obtain cardiac views in a routine NT scan, specifically in centres with high patient load and turnover. Under these usual conditions, our aim was to know whether it is 
possible to give some extra 5-10 minutes in the routine NT scan in order to obtain the 4 standard cardiac views, in an unselected obstetric population.

This combined EFEC-NT (nuchal translucency) approach (11 weeks - 13 weeks +6 days) is reported to detect about $60-90 \%$ of CHD in high risk cases in the hands of experts. $^{3}$ Combined EFEC-NT analysis is justified as CHD is one of the commonest fetal anomaly, there is a high CHD frequency in chromosomal abnormalities/ genetic syndromes and the similarity of anatomic relations between cardiac structures at 11-13 weeks +6 days gestational age and those of the second trimester.

If EFECs are performed for only those with increased NT and other risk factors, majority of cardiac malformations will still be missed as total number of pregnancies with high risk factors are still small and majority of CHDs occur in low risk pregnancies. Hence it makes sense to train all sonographers performing NT scan so that they make an attempt to obtain basic cardiac views at NT scan.

Persico et al have reported the feasibility and accuracy of performing EFEC between 11-13 weeks using high frequency linear TAS by well-trained obstetricians. ${ }^{4}$ In this study obstetricians stored digital clips of cardiac views, interpreted them and also later the digital clips were analysed by specialist fetal cardiologist. Views were inadequate for opinion in only $2.1 \%$ of cases, hence feasibility of obtaining cardiac views by obstetricians is $98 \%$ in this study which is very high. Thus, if obstetricians are trained in obtaining cardiac views during a routine TAS for NT scan, we can achieve good cardiac views after achieving a certain level of training.

\section{Feasibility of obtaining cardiac views in EFEC}

\section{All views/complete cardiac examination}

Feasibility of obtaining all 4 cardiac views seems very modest $(18 \%)$ in the present study compared to highly successful EFEC reported on high risk population by dedicated fetal cardiologist. However, this is the real-life situation during NT scan in most tertiary centres with high patient turnover where only 5-10 extra minutes can be given to EFEC along with NT scan. Thus, performing a complete cardiac examination during routine NT scan may not be possible, unless we can give a minimum of 20-30 extra minutes for the purpose.

A dedicated EFEC performed on high risk patients can achieve very high success in complete cardiac examination, quoted as $88 \%$ at $11-12$ weeks and $99 \%$ in 13-14 weeks. $^{5}$

A dedicated EFEC done on patients referred with risk factors such as increased NT, would be done taking a reasonable amount of time. Such elaborate EFEC can achieve close to $100 \%$ success in obtaining all the cardiac views. $^{6,7}$

\section{Specific views}

Four chamber view has been the easiest to obtain and hence most successful whereas the other views are difficult to obtain. ${ }^{5}$ Most important cardiac views are 4 chamber and 3 vessel views. Out of two at least one is abnormal in a significant number of cardiac anomalies. In our study, 4 chamber view and 3 vessel views were successfully obtained in $79 \%$ and $68 \%$ of the cases, which is satisfactory considering that only 5-10 extra minutes could be devoted to try and obtain cardiac views in these scans. Thus, it would be possible to diagnose a significant number of cardiac anomalies in EFEC done along with routine NT scan. It is noteworthy that obtaining LVOT and RVOT views were possible in only $47 \%$ and $28 \%$ of the cases. This means obtaining these views generally needs a dedicated EFEC session.

None of the cardiac views could be obtained in $16 \%$ of cases wherein there were multiple unfavourable factors for EFEC including obesity, vaginal bleeding hence relative contraindication for TVS, as well as unfavourable fetal position in the limited time available. These factors again mimic the real-life situations in routine NT scanning.

\section{Gestational age versus EFEC}

As shown in Table 2, feasibility of obtaining cardiac views increases as gestational age approaches 14 weeks. A prospective cross-sectional study on early fetal echo analysed the feasibility of EFEC views at various gestational ages. Complete evaluation of the fetal heart was impossible at 10 weeks; the total success rate increased from $45 \%$ at 11 weeks to $90 \%$ between 12 and 14 weeks and $100 \%$ at 15 weeks. ${ }^{6}$ Accuracy of early anatomical survey is high at 12 weeks. Accuracy of aneuploidy screening would be lower as we approach 14 weeks. If we want to obtain the benefit of all these modalities, 12-13 weeks seems to be the best gestational age for EFEC in an unselected obstetric population.

\section{TVS versus TAS}

A prospective cross-sectional study on EFEC analysed relative efficacy of TAS and TVS for EFEC. ${ }^{6}$ Between 10 and 13 weeks, TVS was superior to TAS. At 14 weeks, both methods were similar to each other, and at 15 weeks, TAS allowed adequate visualization of all structures.

Jodi et al published a series of EFEC by an experienced cardiac scanning team on a high-risk population. ${ }^{2}$ Out of 151 studies (mean age 14.5weeks), transabdominal imaging was sufficient for 104; transvaginal imaging was used in 38. In the present study we found that TVS enhanced the clarity of cardiac views in the majority (76\%) of NT scans between 12 to $13+6$ weeks, although 
it was essential to obtain cardiac views in only $3.6 \%$ of cases at this gestational window. This further supports the use of TVS for early anomaly scans where the quality of imaging can be enhanced for detection of all anomalies, not only cardiac.

\section{Accuracy of EFEC in the diagnosis of cardiac anomalies}

On an average a normal EFEC should exclude up to $60 \%$ of fetal cardiac anomalies, in the hands of an expert. ${ }^{8}$

Zeev et al in their study reported that early attempts at EFEC have achieved correct diagnosis of CHD in six out of total 7 CHDs in high risk population. This study reports poor sensitivity to pick up minor cardiac anomalies. ${ }^{5}$ None of the minor anomalies such as small VSDs and ASD were detected in our study as well.

Some diagnoses of CHDs may be incorrect in EFEC, only to reveal a normal fetal heart in later FE. Certain other cardiac diagnoses may be refined later. These problems still exist and must be conveyed to the couple especially high-risk population.

As early as 2005, retrospective as well as prospective studies on EFEC on high risk population have shown the sensitivity of $70 \%$, specificity $98 \%$, positive predictive value $87.5 \%$, and negative predictive value $96 \%$. $^{7}$

A retrospective study on the accuracy of EFEC performed in 2006 reported a pick-up rate of $63 \%$ (29 out of 46 CHDs were picked up at 11-13+6 scan as against the postnatal diagnosis). ${ }^{6}$ However recent studies have coated much higher accuracy. Ventriglia et al in their study quote very high accuracy of EFEC in detecting cardiac anomalies, which was very close to that of late fetal echo. As compared to late fetal echo, Sensitivity was $91.7 \%$, specificity $100 \%$, positive predictive value of of $100 \%$ and negative predictive value of $98.8 \% .^{3}$

As in present study, Mirza et al in his study reported that minor cardiac defects such as small muscular VSDs and isolated persistent left SVC have been missed in the EFEC only to be detected in late EF. ${ }^{9}$ In the same study, a high degree of accuracy was found while detecting major cardiac malformations including AVSD, hypoplastic right heart, TGA, COA etc. At the same time, they reported some false positive findings such as VSD and ventricular disproportion which was not found in late EFEC. As such, this retrospective study on EFEC quoted a sensitivity of $60.0 \%$, specificity of $98.7 \%$, positive predictive value of $75 \%$, and negative predictive value of 97.4\%; as against the standard FE.

Jodi et al reported very high accuracy in diagnosis of CHD with EFEC. ${ }^{2}$ Sensitivity was $100 \%$ as against the standard detection. However, there were some limitations. Three of 19 initially diagnosed with CHD had normal hearts; Seven had modification of original diagnoses: three initially diagnosed with possible septal defects had no defect, two had diagnoses that changed, and two had progression different than predicted. Hence, EFEC as of now does not replace the standard mid trimester fetal echo which is an essential element of pregnancy care.

Persico et al have reported that well trained obstetricians could accurately diagnose $84 \%$ of 100 cardiac anomalies at 11-13 weeks at NT scan using high frequency linear TAS probe. Another 9 cases were suspected to have cardiac anomaly at this EFEC which was later confirmed by fetal cardiologist. Only 5 of 100 cardiac anomalies were completely missed by trained obstetricians at NT scan. ${ }^{4}$ This high level of accuracy can be achieved only after a good amount of training which is feasible in routine practice with good motivation and supervised training. Out of 767 reported "normal" at EFEC, there were 4 major cardiac defects diagnosed at mid trimester FE. ${ }^{4}$ Thus even in the hands of well-trained obstetricians at NT scan, major cardiac defects can be missed hence a follow up mid trimester FE is a must in clinical practice. This must be conveyed to the couple while simultaneously reassuring them that a normal EFEC gives fair degree of reassurance on normal cardiac anatomy in the hands of an expert obstetrician.

The total number of major cardiac anomalies is too small (3) in this study, to comment on the accuracy of EFEC as against standard FE. A case of visceral heterotaxy was missed by EFEC. In this case, EFEC reported that it was difficult to obtain cardiac views and 4 chamber view alone was mentioned. Hence it would be prudent to select those cases where cardiac views are not satisfactorily obtained by EFEC, to a specialist FE either at the same time or at least at 16 weeks.

The sensitivity as well as yield of CHD diagnosis by EFEC is definitely higher among high risk versus unselected obstetric population. This factor should be incorporated in the counselling.

4-D Spatio Temporal Image Correlation (STIC) technology has been used with high accuracy especially using TVS probe for EFEC. Volume acquisition followed by offline analysis resulted in high (95\%) accuracy in diagnosing cardiac anomalies, accuracy was similar to that of conventional 2D+Colour Doppler EFEC in this study. ${ }^{10}$

There are some technical limitations for EFEC which include patient related factors: obesity, CRL $<50 \mathrm{~mm}$, unfavourable fetal position and a possible progression of cardiac disease especially in outflow obstructions.

This means that the pregnant women should be informed about the limitations of early screening and also recommended to have a further scan between 18-22 weeks for a more complete diagnosis. 


\section{CONCLUSION}

It is feasible to get the basic cardiac views in the limited time available to perform NT scan among unselected obstetric population in a tertiary maternity center with a high turnover. However, visualization of outflow tracts and a complete cardiac examination needs a dedicated session for EFEC. Gestational age of 12 weeks and above seems to increase the accuracy. TVS is a useful adjunct throughout 11-14 weeks scan. Although diagnostic accuracy is not high in this study, we propose that making an attempt to obtain cardiac views in the limited time available for NT scan would encourage operators to improve their echocardiography skills over time, thus leading to early diagnosis of fetal cardiac anomalies with a desirable impact on the pregnancy outcome.

Funding: No funding sources

Conflict of interest: None declared

Ethical approval: The study was approved by the Institutional Ethics Committee

\section{REFERENCES}

1. Jonathan K, Shubhika S, Miwa G, John D, Helen K, Jay $\mathrm{S}$, et al. Impact of changing indications and increased utilization of fetal echocardiography on prenatal detection of congenital heart disease. Congenital Heart Dis. 2017;12:67-73.

2. Jodi IP, Anita K, Mary TD. Early fetal echocardiography: congenital heart disease detection and diagnostic accuracy in the hands of an experienced fetal cardiology program. Prenatal Diag. 2014;34:790-6.

3. Ventriglia F, Caiaro A, Giancotti A, Abed MM, Ceccacci I, Celani S, et al. Reliability of early fetal echocardiography for congenital heart disease detection: a preliminary experience and outcome analysis of 102 fetuses to demonstrate the value of a clinical flow-chart designed for at-risk pregnancy management. Pediatr Ther. 2016;6:270.

4. Persico N, Moratalla J, Lombardi CM, Zidere V, Allan L. Fetal echocardiography at 11-13 weeks by transabdominal high frequency ultrasound. Ultrasound Obstet Gynecol. 2011;37:296-301.

5. Zeev W, Abraham L, Eliezer S. Diagnosis of congenital cardiac defects between 11 and 14 weeks' gestation in high-risk patients. J Ultrasound Med. 2002;21:23-9.

6. Jan MS, Christoph B, Annegret G, Rolf F, Roland A, Klaus D, et al. Detection rate of early fetal echocardiography and in utero development of congenital heart defects. J Ultrasound Med. 2006;25:187-96.

7. Mc Auliffe FM, Trines J, Nield LE, Chitayat D, Jaeggi E, Hoenberger LK. Early fetal echocardiography: a reliable prenatal diagnosis tool. Am J Obstet Gynecol. 2005;193:1253-9.

8. Marcin W, Aganieszka N, Jill B. Early fetal echocardiography at the time of $11+0-13+6$ weeks scan. Donald School J Ultrasound Obstet Gynecol. 2009;3(3):75-81.

9. Mirza FG, Bauer ST, Williams IA, Simpson LL. Early fetal echocardiography: ready for prime time? Am J Perinatol. 2012;29(4):313-8.

10. Turan S, Turan OM, Ty-Torredes K, Harman CR, Baschat AA. Department of standardization of the first-trimester fetal cardiac examination using spatiotemporal image correlation with tomographic ultrasound and color Doppler imaging. Ultrasound Obstet Gynecol. 2009;33:652-6.

Cite this article as: Poojari VG, Reddy A, Vasudeva A. Feasibility and accuracy of fetal echocardiography at routine 11 to 13 weeks 6 days ultrasound performed on unselected obstetric population in a tertiary maternity centre with high patient turnover. Int J Reprod Contracept Obstet Gynecol 2018;7:15964. 